\title{
Approximated solutions to Born-Infeld dynamics
}

\author{
Rafael Ferraro ${ }^{a, b, 1}$ and Mauro Nigro ${ }^{b}$ \\ ${ }^{a}$ Instituto de Astronomía y Física del Espacio (IAFE, CONICET-UBA), \\ Casilla de Correo 67, Sucursal 28, 1428 Buenos Aires, Argentina \\ ${ }^{b}$ Departamento de Física, Facultad de Ciencias Exactas y Naturales, Universidad de Buenos Aires, \\ Ciudad Universitaria, Pabellón I, 1428 Buenos Aires, Argentina \\ E-mail: ferraro@iafe.uba.ar, nigro@df.uba.ar
}

ABSTRACT: The Born-Infeld equation in the plane is usefully captured in complex language. The general exact solution can be written as a combination of holomorphic and antiholomorphic functions. However, this solution only expresses the potential in an implicit way. We rework the formulation to obtain the complex potential in an explicit way, by means of a perturbative procedure. We take care of the secular behavior common to this kind of approach, by resorting to a symmetry the equation has at the considered order of approximation. We apply the method to build approximated solutions to Born-Infeld electrodynamics. We solve for BI electromagnetic waves traveling in opposite directions. We study the propagation at interfaces, with the aim of searching for effects susceptible to experimental detection. In particular, we show that a reflected wave is produced when a wave is incident on a semi-space containing a magnetostatic field.

KEYwords: Integrable Equations in Physics, Integrable Field Theories

ArXiv EPrint: 1508.01791

\footnotetext{
${ }^{1}$ Member of Carrera del Investigador Científico (CONICET, Argentina).
} 


\section{Contents}

1 Introduction 1

2 Complex formulation 3

3 Perturbative expansion 5

4 Periodic solutions $\quad 6$

5 The Born-Infeld wave equation $\quad 7$

6 Born-Infeld electromagnetic waves $\quad 7$

6.1 Mirror 8

6.2 Interface with a magnetostatic uniform field 9

$\begin{array}{lll}7 & \text { Conclusions } & 11\end{array}$

\section{Introduction}

Born-Infeld (BI) electrodynamics [1] is a non-linear generalization of Maxwell's theory. BI action governs the tensor field $F_{i j}=\partial_{i} A_{j}-\partial_{j} A_{i}$; it is defined as

$$
\mathcal{S}\left[A_{i}\right]=\frac{b^{2}}{4 \pi} \int d^{4} x\left(1-\sqrt{1+\frac{2 S}{b^{2}}-\frac{P^{2}}{b^{4}}}\right),
$$

where $S$ and $P$ are the scalar and pseudo-scalar

$$
S \equiv \frac{1}{4} F_{i j} F^{i j}=\frac{1}{2}\left(B^{2}-E^{2}\right), \quad P \equiv \frac{1}{4} * F_{i j} F^{i j}=\mathbf{E} \cdot \mathbf{B} .
$$

BI dynamical equations in vacuum are

$$
\partial_{i} \mathcal{F}^{i j}=0, \quad \text { where } \quad \mathcal{F}_{i j} \equiv \frac{F_{i j}-\frac{P}{b^{2}} * F_{i j}}{\sqrt{1+\frac{2 S}{b^{2}}-\frac{P^{2}}{b^{4}}}} .
$$

Constant $b$ in eqs. (1.1) and (1.3) has units of field; it represents the scale for which BI theory departs from Maxwell's theory. In the weak field regime both theories lead to similar results, since $\mathcal{F}^{i j}$ in eq. (1.3) goes to $F^{i j}$ in the limit $b \rightarrow \infty$ (see also References [2-4]).

Although the historical motivation for BI theory was to circumvent the infinite selfenergy of Maxwell's point-like charge (by enforcing the electric field to have the value $b$ at the position of the charge), nowadays the interest in this kind of theory has moved 
towards high-energy physics, since the low energy limit of string theories contains BIlike Lagrangians [5-9]. Except for a case without physical interest, BI theory is the only non-linear electrodynamics free of birefringence and shock waves, and displaying causal propagation [10-14]. Similarities between BI dynamics and MHD equations have been considered in ref. [15].

Apart from the trivial spherically symmetric case, exact stationary BI configurations are hard to obtain; they have been studied in the form of perturbative series $[16,17]$. However, BI electrostatics in the plane can be exactly solved, although in an implicit way. After replacing $\mathbf{E}=-\nabla u(x, y)$ and $\mathbf{B}=0$ in eq. (1.3), one obtains the equation governing the electrostatic potential $u(x, y)$ :

$$
\left(1-b^{-2} u_{, y}^{2}\right) u_{, x x}+2 b^{-2} u_{, y} u_{, x} u_{, y x}+\left(1-b^{-2} u_{, x}^{2}\right) u_{, y y}=0,
$$

Equation (1.4) can be regarded as a non-linear extension of the Laplace equation $\Delta u(x, y)=$ 0 . While the Laplace equation in the plane immediately refers to holomorphic functions, since the real and imaginary parts of any holomorphic function verify Laplace equation, the relation between the solutions to eq. (1.4) and holomorphic functions is much more subtle. This relation was obtained by Pryce [18, 19] (see also References [20-23]). It is worth mention that eq. (1.4) can be also obtained directly from the action

$$
\mathcal{S}[u]=\frac{b^{2}}{4 \pi} \int d x \wedge d y \sqrt{1-b^{-2}\left(u_{, x}^{2}+u_{, y}^{2}\right)} .
$$

This action coincides with the one for the problem of maximal surfaces in (2+1) Minkowski space (space-like surfaces with vanishing mean curvature). It is well known that the solution to this geometrical problem can be written in a parametric way; if $t=b^{-1} u(x, y)$ is the equation for the surface defined on a domain $D$ of the complex plane $z=x+i y$, then the solutions admit the Weierstrass-Enneper parametrization [24]:

$$
(x(\varsigma), y(\varsigma), t(\varsigma))=\operatorname{Re} \int\left(\frac{1}{2} \phi\left(1+\varphi^{2}\right), \frac{i}{2} \phi\left(1-\varphi^{2}\right),-\phi \varphi\right) d \varsigma,
$$

where $\phi$ is holomorphic and $\varphi$ is meromorphic on $D$ such that $\phi \varphi^{2}$ is holomorphic on $D$ and $|\varphi(z)| \neq 1$ for $z \in D$. Like the Weierstrass-Enneper parametrization, also the method of References [18-23] is unable of offering the solutions in an explicit way. Instead, the solution $u(x, y)=u(z, \bar{z})$ is given in an implicit way through a function $z=z(w, \bar{w}), u$ being the real part of $w$.

Equation (1.4) becomes a wave equation by replacing $y$ with it. At the level of the action (1.5), this procedure leads to a descendent of the Nambu-Goto action in $2+1$ dimensions [25], which straightforwardly connects BI wave equation with string theory. Like its static relative (1.4), the Born-Infeld wave equation for the scalar potential $u(t, x)$ also provides solutions to some configurations of BI electrodynamics. We remark that, as a consequence of the non-linearity, BI electromagnetic waves interact with other waves and static fields [26-31]. Only free plane waves evolve like in Maxwell's theory, because $S$ and $P$ vanish in such case (compare with the cylindrical wave in ref. [32]). 
Due to its relevance both for static and propagating BI electromagnetic configurations, together with the impact in related theories, the methods to get solutions to eq. (1.4) are deserving of our attention. The rest of the paper is organized as follows. In section 2 we show that action (1.5) can be transformed into a simple form quadratic in the derivatives, when written in terms of the fields $z=z(w, \bar{w})$. In section 3 we invert the exact linear equation accomplished by $z=z(w, \bar{w})$ to get a first order differential equation for the complex potential $w=w(z, \bar{z})$, which is valid at the first order in $b^{-2}$. In section 4 we solve for periodic $w(z, \bar{z})$ functions, and heal the unbounded behavior characterizing this kind of perturbative approach. The healing method takes advantage of a symmetry of the differential equation at the considered order of approximation. In section 5 we solve the BI scalar wave equation for a configuration of two waves traveling in opposite directions. The interaction between these waves reveals as a modulation of the amplitudes and a shift in the dispersion relation. As shown in section 6 , this solution can be exploited to obtain configurations of equally polarized BI electromagnetic waves traveling in opposite directions; in particular we get the expression for a BI electromagnetic wave interacting with a mirror. We also show that a BI electromagnetic wave entering a region containing a uniform magnetic field is reflected at the interface and changes its polarization; this is an effect typical of a non-linear theory, susceptible of experimental detection. In section 7 we display the conclusions.

\section{Complex formulation}

We will change from Cartesian coordinates to complex coordinates in the plane: $(x, y) \longrightarrow$ $(z, \bar{z})$. So, it follows that

$$
d x^{2}+d y^{2}=|d z|^{2}=d z d \bar{z}
$$

which means that the metric tensor in the chart $(z, \bar{z})$ has the components

$$
g_{z z}=0=g_{\overline{z z}}, \quad g_{z \bar{z}}=\frac{1}{2}=g_{\bar{z} z} .
$$

By replacing $u_{, x}^{2}+u_{, y}^{2}=g^{\mu \nu} u_{, \mu} u_{, \nu}=4 u_{z} u_{\bar{z}}$ (the subindexes indicate partial derivatives) the action (1.5) becomes

$$
\mathcal{S}[u]=\frac{b^{2}}{8 \pi i} \int d z \wedge d \bar{z} \sqrt{1-4 b^{-2} u_{z} u_{\bar{z}}} .
$$

From this action we can reobtain the equation (1.4) written in terms of the derivatives $u_{z}$, $u_{\bar{z}}:$

$$
u_{z \bar{z}}+\frac{1}{b^{2}} u_{\bar{z}}^{2} u_{z z}-\frac{2}{b^{2}} u_{z} u_{\bar{z}} u_{z \bar{z}}+\frac{1}{b^{2}} u_{z}^{2} u_{\overline{z z}}=0 .
$$

The equipotential lines $u(x, y)=$ constant can be regarded as coordinate lines of a nonCartesian chart; so we will introduce a change of coordinates $(z, \bar{z}) \longrightarrow(w, \bar{w})$, where the real and imaginary parts of $w=u+i v$ are coordinates $(u, v)$ in $\mathbb{R}^{2}$. The Jacobian matrix is

$$
\left(\begin{array}{ll}
u_{z} & u_{\bar{z}} \\
v_{z} & v_{\bar{z}}
\end{array}\right)=\left(\begin{array}{cc}
\frac{\partial z}{\partial u} & \frac{\partial z}{\partial v} \\
\frac{\partial \bar{z}}{\partial u} & \frac{\partial \bar{z}}{\partial v}
\end{array}\right)^{-1}=\left(\begin{array}{cc}
\left(z_{w}+z_{\bar{w}}\right) & i\left(z_{w}-z_{\bar{w}}\right) \\
\left(\bar{z}_{w}+\bar{z}_{\bar{w}}\right) & i\left(\bar{z}_{w}-\bar{z}_{\bar{w}}\right)
\end{array}\right)^{-1}
$$


This coordinate change is not unique, since we are free to choose the coordinate $v$. We are going to show that BI equation (2.4) is remarkably simple when written not for $u(z, \bar{z})$ but for $z(w, \bar{w})$, provided a convenient coordinate $v$ is chosen. Let us rewrite the pieces entering the action (2.3). On the one hand it is

$$
d z \wedge d \bar{z}=\left(z_{w} \bar{z}_{\bar{w}}-z_{\bar{w}} \bar{z}_{w}\right) d w \wedge d \bar{w} .
$$

On the other hand we can solve $u_{z}$ from eq. (2.5):

$$
u_{z}=\frac{1}{2} \frac{\bar{z}_{w}-\bar{z}_{\bar{w}}}{z_{\bar{w}} \bar{z}_{w}-z_{w} \bar{z}_{\bar{w}}} .
$$

Therefore

$$
d z \wedge d \bar{z} \sqrt{1-4 b^{-2} u_{z} u_{\bar{z}}}=d w \wedge d \bar{w} \sqrt{\left(z_{\bar{w}} \bar{z}_{w}-z_{w} \bar{z}_{\bar{w}}\right)^{2}-b^{-2}\left(\bar{z}_{w}-\bar{z}_{\bar{w}}\right)\left(z_{\bar{w}}-z_{w}\right)} .
$$

Since $u(z, \bar{z})$ is the sole degree of freedom in the action (2.3), we are free to choose the partner coordinate $v(z, \bar{z})$ in the most convenient way. So, we can look for a choice that simplifies the result (2.8). Then, we will choose the complex coordinate $w=u(z, \bar{z})+i v(z, \bar{z})$ in such a way that the vector $\partial / \partial w$ gets the form

$$
2 b \frac{\partial}{\partial w}=\xi \frac{\partial}{\partial z}+\frac{1}{\xi} \frac{\partial}{\partial \bar{z}}
$$

for some auxiliary function $\xi$. Thus

$$
\frac{\partial z}{\partial w}=\frac{\xi}{2 b}, \quad \frac{\partial z}{\partial \bar{w}}=\frac{1}{2 b \bar{\xi}} .
$$

Our choice for $w(z, \bar{z})$ implies that $(u, v)$ are orthogonal coordinates. In fact,

$$
\begin{aligned}
\frac{\partial}{\partial u} \cdot \frac{\partial}{\partial v} & =i\left(\frac{\partial}{\partial w}+\frac{\partial}{\partial \bar{w}}\right) \cdot\left(\frac{\partial}{\partial w}-\frac{\partial}{\partial \bar{w}}\right)=i \frac{\partial}{\partial w} \cdot \frac{\partial}{\partial w}-i \frac{\partial}{\partial \bar{w}} \cdot \frac{\partial}{\partial \bar{w}} \\
& =i\left(g_{w w}-g_{\overline{w w}}\right)=2 i g_{z \bar{z}}\left(\frac{\partial z}{\partial w} \frac{\partial \bar{z}}{\partial w}-\frac{\partial z}{\partial \bar{w}} \frac{\partial \bar{z}}{\partial \bar{w}}\right)=\frac{i}{4 b^{2}}\left(\frac{\xi}{\xi}-\frac{\bar{\xi}}{\bar{\xi}}\right)=0 .
\end{aligned}
$$

The form of $z_{w}, z_{\bar{w}}$ given in eq. (2.9) is replaced in (2.8) to obtain

$d z \wedge d \bar{z} \sqrt{1-4 b^{-2} u_{z} u_{\bar{z}}}=\frac{d w \wedge d \bar{w}}{4 b^{2}}\left(\frac{1}{|\xi|^{2}}-2+|\xi|^{2}\right)=d w \wedge d \bar{w}\left(\left|\frac{\partial z}{\partial \bar{w}}\right|^{2}-2+\left|\frac{\partial z}{\partial w}\right|^{2}\right)$.

This simple action leads to trivial dynamical equations,

$$
\frac{\partial^{2} z}{\partial w \partial \bar{w}}=0,
$$

which says that $z(w, \bar{w})$ is the sum of a holomorphic function of $w$ and an anti-holomorphic function. Notice that the Born-Infeld constant $b$ is absent in eq. (2.12). Actually this equation is satisfied by Maxwellian solutions too. However, the derivatives of $z(w, \bar{w})$ are 
connected by the eq. (2.9), which links the holomorphic and the anti-holomorphic sectors; this is the only role left to $b$. Therefore the general solution is

$$
z=f(w)+\frac{g(\bar{w})}{4 b^{2}}
$$

(the factor $4 b^{2}$ has been chosen for convenience), where functions $f$ and $g$ are linked by the condition

$$
\overline{f^{\prime}(w)} g^{\prime}(\bar{w})=1 .
$$

Ideally, the potential $u(z, \bar{z})=\operatorname{Re}[w(z, \bar{z})]$ would be obtained by inverting the result (2.13). However, we can hardly solve $w(z, \bar{z})$ from eq. (2.13), except for trivial choices for $f$ that could be deprived of physical interest.

\section{Perturbative expansion}

The inversion of eq. (2.13) can be approached in a perturbative way. For $b^{2}$ going to infinity we recover the Maxwellian result (the complex potential is an analytic function of $z$ ). Then we can start from a known Maxwellian solution $z=f(w)$ and solve the equations (2.13) and (2.14) at the order $b^{-2}$; thus we will find the Born-Infeld corrections at the lowest order. At the level of eq. (2.4), this approach is equivalent to replace the Maxwellian potential in the three terms containing the factor $b^{-2}$.

So we will now focus on getting a differential equation for $w(z, \bar{z})$, at the first order in $b^{-2}$, that can be easily integrable. By differentiating eq. (2.13) with respect to $z$ and $\bar{z}$ we get two equations,

$$
\begin{aligned}
& f^{\prime}(w) \frac{\partial w}{\partial z}+\frac{g^{\prime}(\bar{w})}{4 b^{2}} \frac{\partial \bar{w}}{\partial z}=1, \\
& f^{\prime}(w) \frac{\partial w}{\partial \bar{z}}+\frac{g^{\prime}(\bar{w})}{4 b^{2}} \frac{\partial \bar{w}}{\partial \bar{z}}=0 .
\end{aligned}
$$

Let us conjugate the eq. (3.2) to solve $\partial \bar{w} / \partial z$,

$$
\frac{\partial \bar{w}}{\partial z}=-\frac{1}{4 b^{2}} \frac{\overline{g^{\prime}(\bar{w})}}{\overline{f^{\prime}(w)}} \frac{\partial w}{\partial z} .
$$

Therefore, at the first order in $b^{-2}$, only the first term in eq. (3.1) is relevant. Then it follows that

$$
f^{\prime}(w) \frac{\partial w}{\partial z} \simeq 1
$$

But eq. (2.13) means that $f^{\prime}(w)=\partial z / \partial w$. Therefore, eq. (3.4) becomes

$$
\frac{\partial z}{\partial w} \frac{\partial w}{\partial z} \simeq 1
$$

Eq. (3.5) is not trivial, since the potential $w$ is a function of $z$ and $\bar{z}$. It is only trivial in Maxwell's theory, where the potential is analytic in $z$. Eq. (3.5) means that such a 
Maxwellian property is also guaranteed in Born-Infeld theory at order $b^{-2}$. In eq. (3.4) we can solve $f^{\prime}(w)$ in terms of $\partial w / \partial z$. Thus, eq. (2.14) gives $g^{\prime}(\bar{w})$ in terms of $\partial \bar{w} / \partial \bar{z}$,

$$
g^{\prime}(\bar{w})=\frac{1}{\overline{f^{\prime}(w)}} \simeq \frac{\partial \bar{w}}{\partial \bar{z}}
$$

Results (3.4) and (3.6) are used in eq. (3.2) for getting the differential equation

$$
\frac{\partial w}{\partial \bar{z}}+\frac{1}{4 b^{2}} \frac{\partial w}{\partial z}\left(\frac{\partial \bar{w}}{\partial \bar{z}}\right)^{2}=\mathcal{O}\left(b^{-4}\right) \text {. }
$$

\section{Periodic solutions}

In eq. (3.7), let us try the solution

$$
w(z, \bar{z})=D \cos [k z+\Psi(\bar{z})], \quad k \in \mathbb{R},
$$

which is associated with the Maxwellian seed $w(z)=D \cos (k z)$ describing a periodic configuration. In the second term of eq. (3.7), we replace $w$ with its Maxwellian value, without error at the considered order. Then, one obtains

$$
\Psi^{\prime}(\bar{z})+\frac{k^{3} \bar{D}^{2}}{4 b^{2}} \sin ^{2}(k \bar{z})=0
$$

Thus the complex potential is

$$
w(z, \bar{z})=D \cos \left[k z-\frac{k^{3} \bar{D}^{2}}{8 b^{2}} \bar{z}+\frac{k^{2} \bar{D}^{2}}{16 b^{2}} \sin [2 k \bar{z}]\right]+\mathcal{O}\left(b^{-4}\right),
$$

which can be simplified by just keeping terms of order $b^{-2}$ in the development of the cosine function,

$$
w(z, \bar{z}) \simeq D \cos \left[k z-\frac{k^{3} \bar{D}^{2}}{8 b^{2}} \bar{z}\right]-\frac{k^{2} D \bar{D}^{2}}{16 b^{2}} \sin [2 k \bar{z}] \sin \left[k z-\frac{k^{3} \bar{D}^{2}}{8 b^{2}} \bar{z}\right] .
$$

This solution becomes Maxwellian in the limit $k^{2} D^{2} \ll b^{2}$. In spite of appearances, the approximated solution (4.4) is not entirely satisfactory because the terms of order $b^{-4}$ in the r.h.s. of eq. (3.7) will include an unbounded term that is linear in $z$. Thus the obtained solution is valid only for small values of $|z|$. This is a common problem in perturbative expansions of solutions to differential equations. In Born-Infeld theory this problem can be solved by resorting to a symmetry displayed by the equation at the lowest order in $b^{-2}$. Since $\partial w / \partial z$ only appears in eq. (3.7) at the order $b^{-2}$, then the replacement

$$
\bar{z} \longrightarrow \bar{z}+\frac{\alpha}{b^{2}} z
$$

would not have consequences for the solution at the considered order. Moreover, by choosing $\alpha=-D^{2} k^{2} / 8$ we obtain the potential

$w(z, \bar{z}) \simeq D \cos \left[\left(1+\frac{k^{4} D^{2} \bar{D}^{2}}{64 b^{4}}\right) k z-\frac{k^{3} \bar{D}^{2}}{8 b^{2}} \bar{z}\right]-\frac{k^{2} D \bar{D}^{2}}{16 b^{2}} \sin \left[2 k\left(\bar{z}-\frac{k^{2} D^{2}}{8 b^{2}} z\right)\right] \sin \left[k z-\frac{k^{3} \bar{D}^{2}}{8 b^{2}} \bar{z}\right]$, 
which satisfies

$$
\frac{\partial w}{\partial \bar{z}}+\frac{1}{4 b^{2}} \frac{\partial w}{\partial z}\left(\frac{\partial \bar{w}}{\partial \bar{z}}\right)^{2}=\frac{k^{5} D \bar{D}^{2}}{128 b^{4}} \cos [k z] \sin [2 k \bar{z}]\left(\bar{D}^{2} \cos [2 k \bar{z}]-4 D^{2} \sin ^{2}[k z]\right)+\mathcal{O}\left(b^{-6}\right) .
$$

The terms of order $b^{-4}$ in the r.h.s. are bounded; so the solution (4.6) is satisfactory. Therefore, even if we are looking for solutions at the lowest order in $b^{-2}$, we have to consider the behavior at the next order to avoid unbounded contributions in the differential equation. The real part of potential (4.6) is then a proper solution to the equation (1.4) at the lowest order in $b^{-2}$.

\section{The Born-Infeld wave equation}

Equation (1.4) becomes a non-linear wave equation by replacing $y$ with $-i t$. Because of this reason, the periodic solution (4.6) can help to find solutions to the BI wave equation

$$
\left(1+b^{-2} u_{, t}^{2}\right) u_{, x x}-2 b^{-2} u_{, t} u_{, x} u_{, t x}-\left(1-b^{-2} u_{, x}^{2}\right) u_{, t t}=0 .
$$

Notice that the real part of the Maxwellian seed is

$$
\operatorname{Re}[D \cos (k z)]=D_{1} \cos (k x) \cos (i k y)-i D_{2} \sin (k x) \sin (i k y) .
$$

If $D_{2}=0$, then the replacement $y \longrightarrow-i$ leads to a stationary wave of amplitude $D_{1}$. But in general the result of replacing $y \longrightarrow-i t$ will not be real. However, by replacing $D_{1}=$ $a_{1}+a_{2}$ and $D_{2}=i\left(a_{2}-a_{1}\right)$ we obtain two waves of amplitudes $a_{1}, a_{2}$ that propagate towards increasing and decreasing values of $x$ respectively. We will now start from the potential (4.6) and follow this recipe to obtain the corresponding BI solution at the order $b^{-2}$. The result is

$$
\begin{aligned}
& u(t, x)=\left\{a_{1} \cos \left[\left(1-\frac{a_{2}^{2} k^{2}}{2 b^{2}}\right) k x-\left(1+\frac{a_{2}^{2} k^{2}}{2 b^{2}}\right) k t\right]+a_{2} \cos \left[\left(1-\frac{a_{1}^{2} k^{2}}{2 b^{2}}\right) k x+\left(1+\frac{a_{1}^{2} k^{2}}{2 b^{2}}\right) k t\right]\right\} \\
& \left\{1-\frac{a_{1} a_{2} k^{2}}{2 b^{2}} \sin \left[\left(1-\frac{a_{2}^{2} k^{2}}{2 b^{2}}\right) k x-\left(1+\frac{a_{2}^{2} k^{2}}{2 b^{2}}\right) k t\right] \sin \left[\left(1-\frac{a_{1}^{2} k^{2}}{2 b^{2}}\right) k x+\left(1+\frac{a_{1}^{2} k^{2}}{2 b^{2}}\right) k t\right]\right\},(5.3)
\end{aligned}
$$

which satisfies the Born-Infeld wave equation at first order in $b^{-2}$ :

$$
\begin{aligned}
& \left(1+b^{-2} u_{, t}^{2}\right) u_{, x x}-2 b^{-2} u_{, t} u_{, x} u_{, t x}-\left(1-b^{-2} u_{, x}^{2}\right) u_{, t t}=-\frac{a_{1} a_{2} k^{6}}{4 b^{4}}(\cos [2 k t]-\cos [2 k x]) \\
& \left\{a_{2}\left(4 a_{1}^{2}+a_{2}^{2}\right) \cos [k(t+x)]+a_{1}\left(4 a_{2}^{2}+a_{1}^{2}\right) \cos [k(t-x)]+a_{1}^{3} \cos [3 k(t-x)]+a_{2}^{3} \cos [3 k(t+x)]\right. \\
& \left.+6 a_{1} a_{2}^{2}(\cos [k(3 t+x)]+\cos [k(t+3 x)])+6 a_{1}^{2} a_{2}(\cos [k(3 t-x)]+\cos [k(t-3 x)])\right\}+\mathcal{O}\left(b^{-6}\right)
\end{aligned}
$$

(notice that a bounded function of order $b^{-4}$ is left).

\section{Born-Infeld electromagnetic waves}

The Born-Infeld wave equation (5.1) is involved in some configurations of BI electrodynamics. For instance, consider the potential

$$
A_{i}=(0,0, u(t, x), 0) .
$$


Then the components of the field tensor are

$$
F_{t y}=E_{y}=\frac{\partial u}{\partial t}, \quad F_{x y}=-B_{z}=\frac{\partial u}{\partial x},
$$

so $P=0$. Therefore the field $\mathcal{F}^{i j}$ is

$$
\mathcal{F}^{i j}=\frac{F^{i j}}{\sqrt{1+\frac{2 S}{b^{2}}}}=\frac{F^{i j}}{\sqrt{1+\frac{1}{b^{2}}\left[\left(\frac{\partial u}{\partial x}\right)^{2}-\left(\frac{\partial u}{\partial t}\right)^{2}\right]}},
$$

and eq. (1.3) becomes the eq. (5.1) for the potential $u(t, x)$. Therefore, the solution (5.3) can be regarded as the corresponding BI electromagnetic configuration for the Maxwell field of two equally polarized monochromatic plane waves propagating along the $x$-axis in opposite directions. Such a configuration could be produced when a plane wave is perpendicularly incident on a plane interface. Since a source distribution will appear at the interface, we remark that the dynamical equation (1.3) for the coupling $A_{j} j^{j}$ becomes

$$
\partial_{i} \mathcal{F}^{i j}=4 \pi j^{j} .
$$

\subsection{Mirror}

If the interface is a mirror, then the electric field must vanish on the mirror. This boundary condition is achieved for $a_{1}=-a_{2} \equiv a$ (assuming that the mirror is located at $x=0$ ). In fact, the potential becomes

$$
\begin{aligned}
u= & 2 a \sin \left[\left(1+\frac{a^{2} k^{2}}{2 b^{2}}\right) k t\right] \sin \left[\left(1-\frac{a^{2} k^{2}}{2 b^{2}}\right) k x\right] \\
& \times\left\{1+\frac{a^{2} k^{2}}{4 b^{2}}\left(\cos \left[\left(1+\frac{a^{2} k^{2}}{2 b^{2}}\right) 2 k t\right]-\cos \left[\left(1-\frac{a^{2} k^{2}}{2 b^{2}}\right) 2 k x\right]\right)\right\} .
\end{aligned}
$$

So, we obtain a stationary wave where $E_{y}=\partial u / \partial t$ has nodes at positions $x_{n}$ given in terms of the arbitrary wavenumber $k$ as follows:

$$
\left(1-\frac{a^{2} k^{2}}{2 b^{2}}\right) k x_{n}=n \pi .
$$

While in Maxwell's theory the consecutive nodes are separated by a half-wavelength, in BI electrodynamics the separation between nodes is affected by the wave amplitude (see References $[29,31]$ for the case of waveguides).

Equation (6.5) also shows that the amplitude of the stationary wave is modulated by its second harmonic. In other words, at the order $b^{-2}$ the field contains the fundamental frequency and its third harmonic. The amplitude of the modulation, $a^{2} k^{2} /\left(4 b^{2}\right)$, is expected to be very weak. This tiny non-linear effect could be evidenced by a sensitive measurement of the electric current in the mirror surface. According to eq. (6.4) a current $j^{y}=g(t) \delta(x)$ is associated with the jump of the magnetic component $\mathcal{F}^{x y}$. Then, it follows that

$$
g(t)=\left.\frac{1}{4 \pi} \Delta \mathcal{F}^{x y}\right|_{x=0}=\frac{a k}{2 \pi} \sin \left[\left(1+\frac{a^{2} k^{2}}{2 b^{2}}\right) k t\right]\left(1-\frac{7 a^{2} k^{2}}{2 b^{2}}+\frac{5 a^{2} k^{2}}{2 b^{2}} \cos \left[\left(1+\frac{a^{2} k^{2}}{2 b^{2}}\right) 2 k t\right]\right) .
$$

This current could be detectable in the walls of the resonant cavities of high power lasers. 


\subsection{Interface with a magnetostatic uniform field}

It is well known that BI electromagnetic waves travel at a speed lower than $c$ (we are using $c=1$ ) in the presence of a magnetostatic field $\mathbf{B}_{o}$. For a propagation direction orthogonal to $\mathbf{B}_{o}$, the propagation velocity is $[10,11,27,28,30]$

$$
\beta=\frac{1}{\sqrt{1+\frac{B_{o}^{2}}{b^{2}}}} .
$$

In this sense, the presence of a magnetostatic field $\mathbf{B}_{o}$ makes the space behave like a refractive medium. Therefore it could be expected that an interface with an (otherwise) empty semi-space containing a uniform magnetostatic field $\mathbf{B}_{o}$ will produce a transmitted wave together with a reflected wave. Notice that Maxwell's theory does not produce any reflection at the interface; since the theory is linear there is no interaction between fields. So the Maxwell wave is not affected by the magnetostatic field; $\mathbf{B}_{o}$ just implies a jump of the magnetic field at the interface, which is governed by a constant surface current perpendicular to $\mathbf{B}_{o}$. These features are not so simple in non-linear Born-Infeld electrodynamics. In fact, the field is governed by $d F=d(d A)=0$ and the eq. (6.4). The last relates the charges and currents on the interface to the jumps of the normal electric and tangential magnetic components of $\mathcal{F}$. The former implies the continuity of the tangential electric and normal magnetic components of $F$. However, differing from Maxwell's theory, $\mathcal{F}$ and $F$ do not coincide; the electric (magnetic) part of $\mathcal{F}$ contains traces of the magnetic (electric) part of $F$ (see the definition of $\mathcal{F}$ in eq. (1.3)). As we will see, this feature is the key to produce a reflected wave at the interface.

We will consider a normally incident BI plane wave on an interface at $x=0$. The region $x>0$ (right region) contains a magnetostatic field $B_{o} \widehat{\mathbf{y}}$; so, we propose a constant surface current $\mathbf{j}=g \delta(x) \widehat{\mathbf{z}}$ at the interface. Since the field $F$ is purely tangential at the interface, the solution must satisfy the continuity of $F_{t y}, F_{t z}$ and $\mathcal{F}_{x y}$; besides the jump of $\mathcal{F}_{x z}$ is governed by the surface current:

$$
\left[\mathcal{F}_{\text {right }}^{x z}-\mathcal{F}_{\text {left }}^{x z}\right]_{x=0}=4 \pi g
$$

In the left region $(x<0)$ the form the field is an incident plane wave polarized along $y$, corrected by a reflected wave polarized along $z$ :

$$
F_{\text {left }}=-E(x-t) d(x-t) \wedge d y+b^{-2} H(x+t) d(x+t) \wedge d z
$$

This means that the incident-reflected solution is not related to the potential (5.3), since the solution (5.3) describes equally polarized incident and reflected waves. However, as the reflected wave in the field (6.10) is already of order $b^{-2}$, the approximated approach will be rather simple in this case.

In the right region, the transmitted wave polarized along $y$ is corrected by a transmitted wave polarized along $z$. Both of them propagate at the speed $\beta$ :

$$
F_{\text {right }}=-B(x-\beta t) d(x-\beta t) \wedge d y+B_{o} d x \wedge d z+b^{-2} K(x-\beta t) d(x-\beta t) \wedge d z .
$$


The equation $d F=0$ is trivially fulfilled in both regions. The continuity of $F_{t y}$ and $F_{t z}$ implies

$$
\left.E\right|_{x=0}=\left.\beta B\right|_{x=0},\left.\quad H\right|_{x=0}=-\left.\beta K\right|_{x=0} .
$$

As will be shown, the corrections $H$ and $K$ cannot vanish, but they are needed to satisfy the eq. (6.9).

Let us compute the field $\mathcal{F}$ as defined in eq. (1.3). Notice that $H$ and $K$ contribute to $b^{-2} P$ and $\left(1+b^{-2} 2 S-b^{-4} P\right)^{-1 / 2}$ at the order $b^{-4}$; thus it follows that

$$
\begin{aligned}
b^{-2} P_{\text {left }} & =\mathcal{O}\left(b^{-4}\right), & \left(1+b^{-2} 2 S-b^{-4} P\right)_{\text {left }}^{-1 / 2}=1+\mathcal{O}\left(b^{-4}\right), \\
b^{-2} P_{\text {right }} & =b^{-2} \beta B_{o} B(x-\beta t)+\mathcal{O}\left(b^{-4}\right), & \left(1+b^{-2} 2 S-b^{-4} P\right)_{\text {right }}^{-1 / 2}=\beta+\mathcal{O}\left(b^{-4}\right) .
\end{aligned}
$$

Then $\mathcal{F}_{\text {left }} \simeq F_{\text {left }}$, and ${ }^{1}$

$$
\begin{aligned}
\mathcal{F}_{\text {right }} & \simeq \beta\left(F-b^{-2} P * F\right)_{\text {right }} \\
& \simeq B d(t-\beta x) \wedge d y+\beta B_{o} d x \wedge d z+b^{-2} \beta^{2} B^{2} B_{o} d(t-\beta x) \wedge d z+b^{-2} \beta K d(x-\beta t) \wedge d z .
\end{aligned}
$$

The continuity of $\mathcal{F}_{x y}$ is guaranteed by the eq. (6.12). The eq. (6.9) implies

$$
\left[\beta B_{o}-b^{-2} \beta^{3} B^{2} B_{o}+b^{-2} \beta K-b^{-2} H\right]_{x=0}=4 \pi g .
$$

Because of eq. (6.12), one gets

$$
\left[\beta B_{o}-b^{-2} \beta E^{2} B_{o}-2 b^{-2} H\right]_{x=0}=4 \pi g .
$$

Let us introduce a monochromatic incident wave $E=e \sin [k(x-t)]$. Thus

$$
\beta B_{o}-\frac{1}{2} b^{-2} \beta e^{2} B_{o}(1-\cos [2 k t])-2 b^{-2}[H]_{x=0}=4 \pi g .
$$

Then

$$
\beta B_{o}\left(1-\frac{e^{2}}{2 b^{2}}\right)=4 \pi g
$$

i.e.,

$$
B_{o}\left(1-\frac{B_{o}^{2}+e^{2}}{2 b^{2}}\right) \simeq 4 \pi g
$$

and

$$
\frac{1}{2} b^{-2} \beta e^{2} B_{o} \cos [2 k t]-2 b^{-2}[H]_{x=0}=0 .
$$

Therefore the reflected wave is

$$
b^{-2} H(t+x)=\frac{e^{2} \beta B_{o}}{4 b^{2}} \cos [2 k(x+t)] \simeq b^{-2} \pi e^{2} g \cos [2 k(x+t)] .
$$

According to eq. (6.12), the transmitted wave is given by

$$
B(x-\beta t)=\beta^{-1} e \sin \left[\frac{k}{\beta}(x-\beta t)\right] \quad \text { and } \quad b^{-2} K(x-\beta t)=-\frac{e^{2} B_{o}}{4 b^{2}} \cos \left[\frac{2 k}{\beta}(x-\beta t)\right] .
$$

\footnotetext{
${ }^{1} * \mathcal{F} \simeq B d(x-\beta t) \wedge d z-\beta B_{o} d t \wedge d y-b^{-2} \beta^{2} B^{2} B_{o} d(x-\beta t) \wedge d y+b^{-2} \beta K d(\beta x-t) \wedge d y$. The equation $d * \mathcal{F}=0$, which is equivalent to the dynamical equation (1.3), is trivially satisfied by any differentiable functions $B(x-\beta t), K(x-\beta t)$ because $d(\beta x-t)$ can be replaced by $d(x-\beta t)$ without error at the considered order.
} 


\section{Conclusions}

Exact solutions to the Born-Infeld electrostatic equation (1.4) (or, equivalently, (2.4)) are linked to a pair of holomorphic and anti-holomorphic functions, $f$ and $g$ respectively, subjected to the condition (2.14). Nevertheless the solutions remain expressed in the implicit way (2.13), where the coordinates $z$ are given as functions of the complex potential $w$. This relation can be inverted order by order in powers of $b^{-2}$, to get the (real) potential as a function of the coordinates. This procedure is equivalent to solve the equations order by order; in this case, the equations are satisfied up to terms of higher order in powers of $b^{-2}$. However such terms could develop secular behaviors for large values of the coordinates. We have taken advantage of the symmetry (4.5) to avoid the undesired behavior. The solutions to the scalar equations (1.4) and (5.1) can be applied to obtain simple configurations of Born-Infeld electrodynamics: those where $\mathbf{E}$ and $\mathbf{B}$ are orthogonal and depend just on two coordinates (a third coordinate can always be introduced by means of a Lorentz boost). With this aim, we considered periodic complex potentials for getting a configuration of electromagnetic BI waves traveling in opposite directions (see Reference [26] for a different approach to this problem). In the case of the stationary wave produced by the reflection of an BI electromagnetic wave normally incident on a mirror, we have shown that the separation between nodes becomes dependent on the amplitude of the wave. Besides, both the wave amplitude and the surface current at the mirror are modulated by the second harmonic. These effects could be detected in cavities, and have consequences in the phenomenology of laser-plasmas [33]. We have also studied the propagation of BI electromagnetic waves at the interface with a region containing a magnetostatic field. It is well known that the magnetostatic field alters the propagation velocity, like a sort of refractive index; this property is exploited in some experimental arrays recently proposed [34]. We have shown that the analogy with a refractive index also works to produce a reflected wave at the interface, whose polarization is orthogonal to the one of the incident wave. Irrespective of the technical difficulties in carrying out this experiment, we should keep the idea that it is possible to test BI electrodynamics by searching for optical effects in (non-necessarily homogeneous) static fields; these effects include deviation of rays and contributions of harmonic frequencies.

\section{Acknowledgments}

This work was supported by Consejo Nacional de Investigaciones Científicas y Técnicas (CONICET) and Universidad de Buenos Aires.

Open Access. This article is distributed under the terms of the Creative Commons Attribution License (CC-BY 4.0), which permits any use, distribution and reproduction in any medium, provided the original author(s) and source are credited.

\section{References}

[1] M. Born and L. Infeld, Foundations of the new field theory, Proc. Roy. Soc. Lond. A 144 (1934) 425 [InSPIRE]. 
[2] M. Born and L. Infeld, Foundations of the new field theory, Nature 132 (1933) 1004.

[3] M. Born, Quantum theory of the electromagnetic field, Proc. Roy. Soc. Lond. A 143 (1934) 410 [INSPIRE].

[4] M. Born and L. Infeld, On the quantization of the new field equations. I, Proc. Roy. Soc. Lond. A 147 (1934) 522 [InSPIRE].

[5] E.S. Fradkin and A.A. Tseytlin, Nonlinear electrodynamics from quantized strings, Phys. Lett. B 163 (1985) 123 [inSPIRE].

[6] A. Abouelsaood, C.G. Callan Jr., C.R. Nappi and S.A. Yost, Open strings in background gauge fields, Nucl. Phys. B 280 (1987) 599 [INSPIRE].

[7] R.G. Leigh, Dirac-Born-Infeld action from Dirichlet $\sigma$-model, Mod. Phys. Lett. A 4 (1989) 2767 [INSPIRE].

[8] R.R. Metsaev, M. Rakhmanov and A.A. Tseytlin, The Born-Infeld action as the effective action in the open superstring theory, Phys. Lett. B 193 (1987) 207 [INSPIRE].

[9] A.A. Tseytlin, On non-Abelian generalization of Born-Infeld action in string theory, Nucl. Phys. B 501 (1997) 41 [hep-th/9701125] [INSPIRE].

[10] J. Plebański, Lectures on non linear electrodynamics, Nordita Lecture Notes, Copenhagen Denmark (1968) [INSPIRE].

[11] G. Boillat, Nonlinear electrodynamics-Lagrangians and equations of motion, J. Math. Phys. 11 (1970) 941 [INSPIRE].

[12] S. Deser and R. Puzalowski, Supersymmetric nonpolynomial vector multiplets and causal propagation, J. Phys. A 13 (1980) 2501 [INSPIRE].

[13] I. Bialynicki-Birula, Nonlinear electrodynamics: variations on a theme by Born and Infeld, in J. Lopuszanski's Festschrift, Quantum Theory of Particles and Fields, B. Jancewicz and J. Lukierski eds., World Scientific, Singapore (1983), pg. 31 [INSPIRE].

[14] R. Kerner, A.L. Barbosa and D.V. Gal'tsov, Topics in Born-Infeld electrodynamics, in Proceedings of XXXVII Karpacz Winter School, J. Lukierski and J. Rembielinski eds., Amer. Math. Soc., Providence U.S.A. (2001) [AIP Conf. Proc. 589 (2001) 377] [hep-th/0108026] [INSPIRE].

[15] Y. Brenier, Hydrodynamic structure of the augmented Born-Infeld equations, Arch. Rat. Mech. Anal. 172 (2004) 65.

[16] M.K.H. Kiessling, Convergent perturbative power series solution of the stationary Maxwell-Born-Infeld field equations with regular sources, J. Math. Phys. 52 (2011) 022902 [INSPIRE].

[17] M.K.-H. Kiessling, Some uniqueness results for stationary solutions to the Maxwell-Born-Infeld field equations and their physical consequences, Phys. Lett. A 375 (2011) 3925.

[18] M.H.L. Pryce, The two-dimensional electrostatic solutions of Born's new field equations, Proc. Cambr. Phil. Soc. 31 (1935) 50.

[19] M.H.L. Pryce, On a uniqueness theorem, Proc. Cambr. Phil. Soc. 31 (1935) 625.

[20] R. Ferraro, 2D Born-Infeld electrostatic fields, Phys. Lett. A 325 (2004) 134 [hep-th/0309185] [INSPIRE]. 
[21] R. Ferraro and M.E. Lipchak, Born-Infeld corrections to Coulombian interactions, Phys. Rev. E 77 (2008) 046601 [hep-th/0609141] [INSPIRE].

[22] R. Ferraro, Born-Infeld electrostatics in the complex plane, JHEP 12 (2010) 028 [arXiv: 1007.2651] [INSPIRE].

[23] R. Ferraro, Two-dimensional solutions for Born-Infeld fields, JHEP 08 (2013) 048 [arXiv: 1304.5506] [INSPIRE].

[24] O. Kobayashi, Maximal surfaces in the 3-dimensional Minkowski space $L^{3}$, Tokyo J. Math. 6 (1983) 297.

[25] M. Bordemann and J. Hoppe, The dynamics of relativistic membranes 2. Nonlinear waves and covariantly reduced membrane equations, Phys. Lett. B 325 (1994) 359 [hep-th/9309025] [INSPIRE].

[26] B.M. Barbashov and N.A. Chernikov, The possibility of an oscillatory nature of gravitational collapse, Sov. Phys. JETP 24 (1967) 437.

[27] H.S. Ibarguen, A. García and J. Plebański, Signals in nonlinear electrodynamics invariant under duality rotations, J. Math. Phys. 30 (1989) 2689 [INSPIRE].

[28] M. Novello, V.A. De Lorenci, J.M. Salim and R. Klippert, Geometrical aspects of light propagation in nonlinear electrodynamics, Phys. Rev. D 61 (2000) 045001 [gr-qc/9911085] [INSPIRE].

[29] R. Ferraro, Testing Born-Infeld electrodynamics in waveguides, Phys. Rev. Lett. 99 (2007) 230401 [arXiv:0710.3552] [INSPIRE].

[30] M. Aiello, G. Bengochea and R. Ferraro, Anisotropic effects of background fields on Born-Infeld electromagnetic waves, Phys. Lett. A 361 (2007) 9 [hep-th/0607072] [InSPIRE].

[31] R. Ferraro, Testing nonlinear electrodynamics in waveguides: the effect of magnetostatic fields on the transmitted power, J. Phys. A 43 (2010) 195202 [InSPIRE].

[32] E. Yu. Petrov and A.V. Kudrin, Exact self-similar solutions in Born-Infeld theory, Phys. Rev. D 87 (2013) 087703 [arXiv:1302.4353] [INSPIRE].

[33] D.A. Burton, R.M. G.M. Trines, T.J. Walton and H. Wen, Exploring Born-Infeld electrodynamics using plasmas, J. Phys. A 44 (2011) 095501 [arXiv: 1006. 2246] [INSPIRE].

[34] G.O. Schellstede, V. Perlick and C. Lämmerzahl, Testing non-linear vacuum electrodynamics with Michelson interferometry, Phys. Rev. D 92 (2015) 025039 [arXiv:1504.03159] [INSPIRE]. 